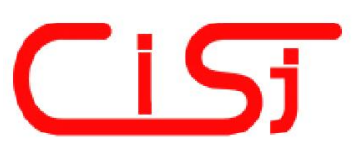

\title{
TECHNIQUE OF CONSTRUCTION MODELS OF BEHAVIOR ALGORITHMS OF RADIO ELECTRONIC COMPLEX SYSTEM USING THE SCHEME OF PATHS METHOD
}

\author{
Bohdan Volochiy, Leonid Ozirkovskyi, Oleksandr Shkiliuk, Andriy Mashchak
}

\author{
Department of Theoretical Radio Engineering and Radio Measurement \\ Lviv Polytechnic National University, \\ S. Bandera Str, 12, Lviv, 79013, Ukraine, \\ e-mail: bvolociy@ukr.net, lozirkovsky@1p.edu.ua,shkiliuk@1p.edu.ua,himakus@yahoo.com
}

\begin{abstract}
This paper presents the technique of constructing models of behavior algorithms of radio electronic complex system using new scheme of paths method. This technique allows estimation of efficiency indexes of Radio Electronic Complex System taking into account behavior algorithm's features, hardware and software reliability and a presence of self-diagnostics procedure. Copyright (C) Research Institute for Intelligent Computer Systems, 2014. All rights reserved.
\end{abstract}

Keywords: behavior algorithm; scheme of paths method; efficiency indexes; radio electronic complex system.

\section{INTRODUCTION}

Radio electronic complex system (RECS) consists of different electronic, information and control subsystems, each of which carries its own set of procedures and is connected with other components of RECS by information network for control and exchanging of all necessary data and service signals. So providing the necessary efficiency indexes of RECS by its behavior algorithm design is very urgent assignment.

The behavior algorithm is formalized representation of RECS information usage while performing its objective function and consists of sequence of procedures. The behavior algorithm is characterized by temporal and functional redundancy and time restrictions for performance. This algorithm describes the functional interrelations between the elements of the system and the functional behavior of the system in general. Such efficiency indexes of RECS as probability of successful performance and mean value of successful performance duration should be determined at the designing stage for reducing the costs of time and material resources.

So it is actual task of parametric synthesis of behavior algorithm of RECS when preset system efficiency index should be distributed to parameters of separate procedures, all of which ensure objective function performance by fixed time.

Behavior algorithm of RECS has following features, which are distinctive for its synthesis and analysis:
- reliability behavior is taken into account in addition to functional behavior of fault-tolerant structures;

- inclusion of self-diagnostics procedures of hardware and software;

- there are two types of conditional units (stochastic alternative and deterministic switching);

- jumps out from the loops by certain cases;

- several end units of behavior algorithm;

- it is embedded as software in control subsystem of RECS.

Presented in this paper scientific achievements were reported at the Dependable Systems, Services \& Technologies conference (DESSERT'2014), which was held in Kyiv, Ukraine in May 16-19, 2014. At this conference the basics of the scheme of paths method for construction mathematical models of behavior algorithm of RECS were represented.

This work is continuation of the paper [1] and includes further researches of behavior algorithm of RECS.

\section{REVIEW OF WELL-KNOWN METHODS FOR CONSTRUCTING MATHEMATICAL MODELS OF THE BEHAVIOR ALGORITHMS}

There are several known methods for analyzing the efficiency indexes of RECS such as logicalprobabilistic method [2-4], simulation modeling $[5,6]$, space of states method [7-10], algorithmic 
algebras method (also known as I.V. Safonov's method) [11], Petri nets [12-14].

\subsection{LOGICAL-PROBABILISTIC METHOD}

The main theoretical approaches and recommendations for constructing logicalprobabilistic models and methodological foundations to formalize them are provided in work [2] as modeling technology. The technology of logical-probabilistic modeling is based on fault tree or event tree. This technology is oriented to analysis of reliability and safety of the automated control systems of aircraft, ground transport, technological processes and control systems of nuclear power plants. In work [3] the technology of logicalprobabilistic modeling was partially automatized. It gives the advantage of significant reduction of development time and the possibility of multivariate analysis of complex systems, but manual construction of the functional integrity scheme, which is the intermediary model of the complex system, is the disadvantage of this modeling technology because the functional integrity scheme upraises to great dimension.

Moreover, standard fault trees are not sufficient in cases when reliability analysis of complex systems must include timing considerations and the order of events. So to express time-dependent behavior fault trees with time dependencies and timed state-charts are used but disadvantage of the method [4] is that determining of Cartesian products that are used in calculation of event duration times and causal gate delay times are not done automatically.

\subsection{SIMULATION}

Simulation allows us to consider the processes occurring in the system almost in any level of details $[5,6]$. In the simulation model almost any algorithm of behavior or control of the system can be implemented, but development of every simulation model is separate task that requires significant time and costs and it is not flexible in modifying the behavior algorithm. Simulation is an alternative method for analyzing the RECS and it is used when experiment is unavailable for developers and researchers.

\subsection{SPACE OF STATES METHOD}

It uses techniques for modeling complex information system and it is based on Markov processes $[7,8]$.

In the monograph [9] analytical technology of modeling the behavior algorithms of information systems, which contains space of states method, is proposed. It presents RECS as discrete-continuous stochastic system, because it allows accomplishing the tasks of definition and studying the performance of RECS. This application requires the creation of structural-automatic model, which is the formal representation of the structure and behavior of RECS. Structural-automatic model is the input data for the software module ASNA, which automatically generates graph of states and transitions, and on its basis forms analytical mathematical model as the system of Chapman - Kolmogorov linear differential equations.

The paper [10] introduces modified space of states method for estimation the efficiency indexes of behavior algorithm of RECS of airspace monitoring such as probability of successful performance and mean time value of successful performance. Development of structural-automatic model involves solving the following tasks: forming the vector of states, forming the set of formal parameters, defining the basic events and forming the tree of modification rules of vector's of states components. Vector of states is the record of all variables that will be used in the forming of tree of modification rules of vector's of states components. Number of vector's of states components defines detailed description of the object in the model. On the other hand the number of vector's of states components component affects the dimension of the model. Vector of states characterizes the current state of each system in every moment time and its mode. For behavior algorithm of RECS only one event takes place - the performance the operational unit. Special requirements are applied to the set of formal parameters, since it must contain all technical and tactical parameters of the RECS (probability of successful performance of procedure, mean time value of its successful performance, reliability of devices and subsystems, operator's response time to messages etc.) that will provide construction of adequate mathematical model of RECS behavior. So developed tree of modification rules of vector's of states represents behavior of RECS.

This method in general takes into account the structure of RECS, its technical and tactical characteristics, subsystems reliability and qualification of the human-operator but the disadvantages of this approach are state space explosion, stiffness and rarefied of system of Chapman - Kolmogorov linear differential equations and the impossibility of normalization mean time value of successful performance of behavior algorithm of RECS to probability of its successful performance.

\subsection{ALGORITHMIC ALGEBRAS METHOD}

In the monograph [11] algorithmic algebras language was selected as basis for modeling the external (functional) and internal (reliability) 
behavior of RECS. Algorithmic algebras language was proposed by V.M. Glushkov for solving problems in cybernetics and programming and it was enhanced by I.V. Safonov for reliability designing of control systems. Algorithmic algebras method uses regular canonical forms of algorithms (linear, disjunctive, iterative and parallel) and can simulate any behavior algorithms or control algorithm of RECS. Model of behavior algorithm of RECS using algorithmic algebras method is formed by setting up the logical expressions, that allows you to get the logical-probabilistic model of behavior algorithm in the form of logical function. For adoption to algorithmic algebras method the behavior algorithm of RECS requires to be represented in tree form, that is significantly time-consuming task, hence changing the structure of the behavior algorithm requires complete restructuring of the model.

\subsection{PETRI NETS}

In practice of modeling objects, when it is needed to solve tasks with formalized description and consideration of cause-and-effect relationships in complex systems, where multiple processes in parallel flows, network approaches such as Petri nets are used [12]. An important feature of the modeling the systems functioning with Petri nets usage is the simplicity of construction of hierarchical structures. Hence it follows possibility of effective Petri nets usage for modeling parallel and competing processes in various systems.

Ordinary Petri nets are used to describe the events of any duration. However, these models reflect only the order of events occurring in the studied system. To display the time parameters of the systems models basing on Petri nets it is need to use different extensions such as colored Petri net [13] or deriving feared scenarios, which might lead the system to critical situation [14]. For complex systems this approach can lead to state space explosion and for avoiding this Petri net reachability is translated into provability of linear logic sequent.

Flowchart of behavior algorithm of RECS can be interpreted in Petri nets by movement of marker (token), which executes instructions. However, the instructions / commands in flowchart are simulated by operating and conditional units and in Petri net by transitions depending on type of unit. Respectively transitions in flowchart are replaced in Petri net to position (place). Modeling the system using Petri nets requires attention to assure that event sequence doesn't occur conflict of two enabled transitions. Then only one transition can remove token and disable other transition. It means that, for example in flowchart of behavior algorithm with loops, the token is ready for execution but the instruction / command, which is associated with certain conditional unit, enters the net in the conflict. Choice of method of solving the conflict is another methodological problem [12]

\subsection{INTERCOMPARISON OF METHODS FOR CONSTRUCTING AND ANALYSIS OF BEHAVIOR ALGORITHMS MODELS}

The results of analysis the possibilities of methods for modeling RECS behavior is presented in Table 1. Thus, only modified space of states method and algorithmic algebras method were originally adopted for efficiency analysis of behavior algorithms of RECS but the analysis of these methods identified some ambiguity concerning the consideration of the loops number and normalization of mean time value of successful performance by the probability of successful performance of RECS.

Table 1. Intercomparison of methods for constructing and analysis of behavior algorithms models.

\begin{tabular}{|l|c|c|c|c|c|}
\hline \multicolumn{1}{|r|}{ methods } & $\begin{array}{c}\text { logical- } \\
\text { probabilistic } \\
\text { method }\end{array}$ & $\begin{array}{c}\text { simulation } \\
\text { modeling }\end{array}$ & $\begin{array}{c}\text { modified } \\
\text { space of } \\
\text { states method }\end{array}$ & $\begin{array}{c}\text { algorithmic } \\
\text { algebras } \\
\text { method }\end{array}$ & $\begin{array}{c}\text { scheme of } \\
\text { paths } \\
\text { method }\end{array}$ \\
\hline $\begin{array}{l}\text { reliability behavior is taken } \\
\text { into account in addition to } \\
\text { functional behavior of fault- } \\
\text { tolerant structures }\end{array}$ & - & + & + & + \\
\hline $\begin{array}{l}\text { inclusion of self-diagnostics } \\
\text { procedures of hardware and } \\
\text { software }\end{array}$ & + & + & + & + & + \\
\hline $\begin{array}{l}\text { there are two types of } \\
\text { conditional units }\end{array}$ & - & + & + & + & + \\
\hline $\begin{array}{l}\text { jumps out from the loops by } \\
\text { certain cases }\end{array}$ & - & - & + & + & + \\
\hline $\begin{array}{l}\text { several end units of behavior } \\
\text { algorithm }\end{array}$ & - & + & + & + \\
\hline
\end{tabular}


For example, algorithmic algebras method, when using regular iterative form, considers unlimited loops number of behavior algorithm, so the probability of successful performance of the algorithm is overrated, but its mean time value is normalized by the probability of successful performance. On the other side, we can use regular disjunctive form of algorithmic algebras method or modified space of states method, which uses absolutely different mathematical tool. Nevertheless, the loops number is strictly defined in these cases, so the probability of successful performance of the algorithm isn't overestimated, but its mean time value is underestimated, because of nonnormalization by the probability of successful performance.

Just so it is the actual problem to develop the method of constructing mathematical models of behavior algorithms for estimation the efficiency indexes of RECS, which would take into account strictly defined loops number and normalization of mean time value of successful performance of behavior algorithm by the its probability of successful performance, that in general will improve the validity of the mathematical model. In addition, flowchart of behavior algorithm should be easily modified to save development time and costs.

\section{TECHNIQUE OF CONSTRUCTION MODELS OF BEHAVIOR ALGORITHMS OF RADIO ELECTRONIC COMPLEX SYSTEMS USING THE SCHEME OF PATHS METHOD}

As the alternative to modeling methods of the behavior algorithms, which were outlined in works $[10,11]$, the scheme of paths method was developed and presented in the paper [1]. Incorporation of features of RECS behavior algorithms in the scheme of paths method is presented in Table 1.

The behavior algorithm consists of operational and conditional units. Operational units reflect the functions of the subsystems, which are a part of the RECS. Duration of each function is random and it is displayed in the model as the mean value of the duration $\mathrm{T}_{\mathrm{m}}$. Conditional units display RECS mode changes during the performance of its objective function.

In the scheme of paths method the separation of conditional units into two types was proposed: stochastic alternative and deterministic switching units. Stochastic alternative unit is characterized by the probability of performance (or non-performance) of system function $(p, q=1-p)$. Deterministic switching unit is characterized by the probability $\mathrm{p}^{+}=1$ if the decision of the unit is true, and the probability $\mathrm{p}^{-}=1$ otherwise. This representation of conditional jumps of deterministic switching units is caused by the requirement to match them with stochastic alternative units. So deterministic switching units can reflect the software scenario and jumps out of the behavior algorithm by case.

This presentation of behavior algorithm is the first step for the construction of its mathematical model in the proposed method. The next step involves the scheme of paths forming. This scheme is formalized representation of the RECS behavior in the compact form that reflects the set of all paths that lead to successful or unsuccessful performance of the objective function. Mathematical model is presented in the form of expression for the longest path from start to successful or unsuccessful performance, taking into account the loops number.

The scheme of paths forming requires converting operational units (OU) into conformed to them functional units (FU) and indicating all transitions between them. The technique of constructing models by scheme of paths method is carried out by the following steps:

- functional unit $\mathrm{FU}_{\mathrm{m}}$ is characterized by the mean value of the duration of the function $\mathrm{T}_{\mathrm{m}}$ and conforms to operational unit $\mathrm{OU}_{\mathrm{m}}$ of the behavior algorithm flowchart;

- if a fragment of the algorithm is repeatedly performed, then it is depicted by repeated functional unit $\mathrm{RFU}_{\mathrm{m}}$, which takes into account parameters of its function re-performance: mean value of the duration $T_{m}$, loops number $n R F U_{m}$ and probability of re-performance $\mathrm{pRF} \mathrm{U}_{\mathrm{m}}$;

- transition parameter $\mathrm{pL}_{\mathrm{m}} \mathrm{m}+1$, which connects functional units $\mathrm{FB}_{\mathrm{m}}$ and $\mathrm{FB}_{\mathrm{m}+1}$ on the scheme of paths, is the product of all probabilities which flow through all stochastic alternative and deterministic switching units that are posessed on the path between the operational units $\mathrm{OU}_{\mathrm{m}}$ and $\mathrm{OU}_{\mathrm{m}+1}$ on the behavior algorithm flowchart;

- several identical end units on the flowchart of behavior algorithm are united into one end functional unit on the scheme of paths.

Guiding by these rules let's form the scheme of paths for behavior algorithm of RECS for searching, detection and locking-on targets (Fig. 1) and write down formula for estimation its efficiency indexes probability and mean time value of successful performance (Eq. 1, 2):

$$
\begin{gathered}
p_{k}\left(S_{S P}\right)=p L_{12} \cdot\left(p R F U_{2}\right)^{n R F U_{2}} \cdot p L_{23} \cdot p L_{3 S}, \\
T_{k}\left(S_{S P}\right)=T_{1}+\left(n R F U_{2}+1\right) \cdot T_{2}+T_{3} .
\end{gathered}
$$

In general, we obtain formulas for estimation the efficiency indexes of RECS for any behavior algorithm as Eq. 3, 4: 


$$
\begin{gathered}
p\left(S_{S P}\right)=\sum_{k=1}^{K}\left(p_{k}\left(S_{S P}\right)\right), \\
T\left(S_{S P}\right)=\frac{1}{p\left(S_{S P}\right)} \sum_{k=1}^{K}\left(p_{k}\left(S_{S P}\right) \cdot T_{k}\left(S_{S P}\right)\right) .
\end{gathered}
$$

As we can see (Eq. 4) mean time value of successful performance of behavior algorithm $\mathrm{T}\left(\mathrm{S}_{\mathrm{SP}}\right)$ is normalized by the its probability of successful performance $\mathrm{p}\left(\mathrm{S}_{\mathrm{SP}}\right)$, that improves the validity of the mathematical model.
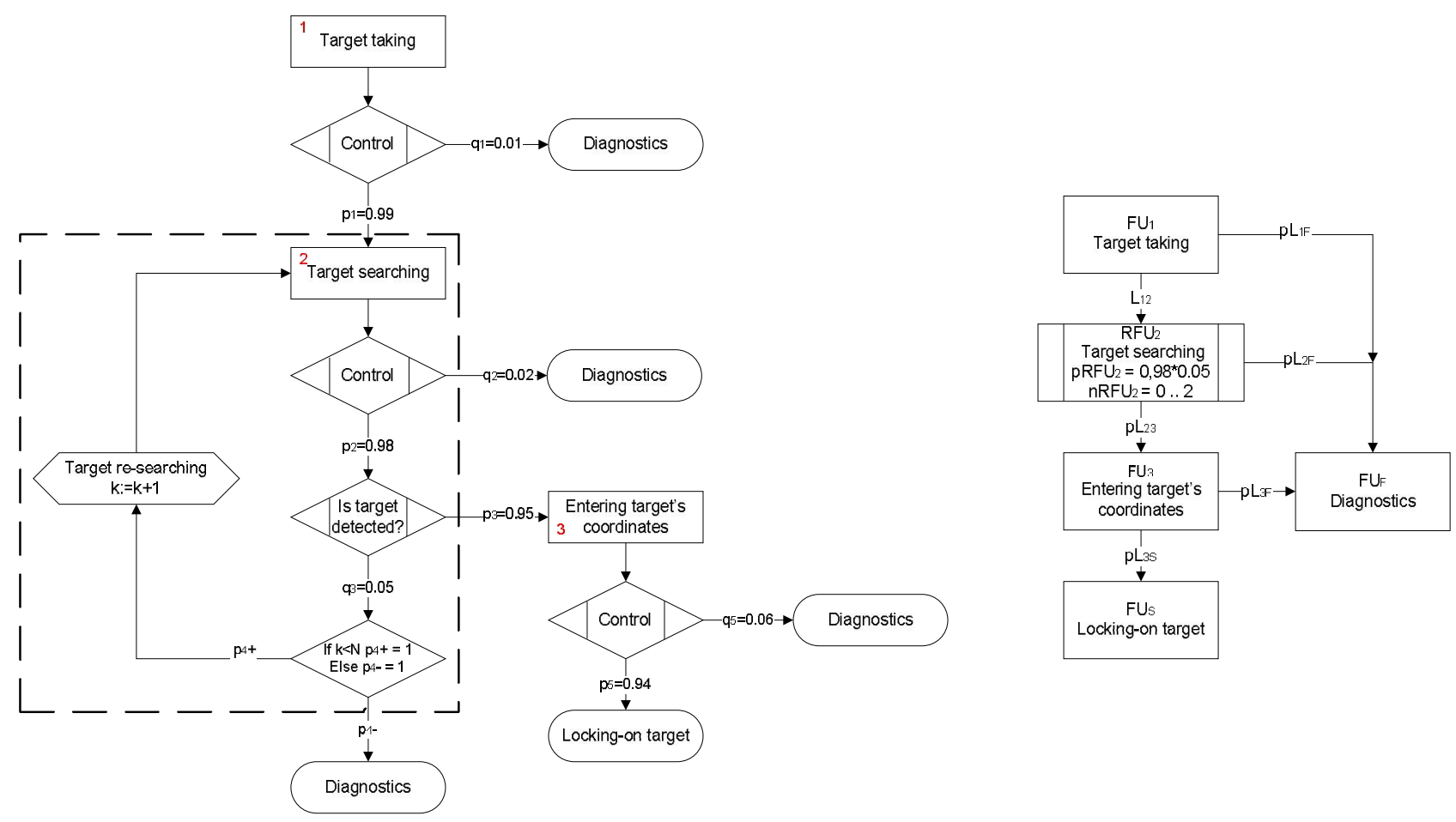

Fig. 1 - Scheme of paths forming from RECS behavior algorithm flowchart.

\section{PRESENTATION OF CAPABILITIES OF THE SCHEME OF PATHS METHOD FOR RESEARCHING THE EFFICIENCY INDEXES OF RADIO ELECTRONIC COMPLEX SYSTEM FOR SEARCHING, DETECTION AND LOCKING-ON TARGETS}

The scheme of paths method was adopted for modeling the behavior algorithm of RECS for searching, detection and locking-on targets. This approach provides efficiency indexes estimation, researching and analysis of RECS for searching, detection and locking-on targets. Such efficiency indexes of this system as high probability and short mean time value of successful performance are required.

The behavior algorithm of the RECS for searching, detection and locking-on targets includes different functions and procedures. The specific sequence of their performances ensures the complex system's objective function.

The behavior algorithm of the RECS for searching, detection and locking-on targets involves next functions and procedures: target taking, target searching, target re-searching, control, diagnostics, entering target's coordinates, locking-on target (Fig. 1). For each particular synthesis task the probability of successful performance and mean time value of successful performance can be estimated depending on the impact of hardware and software, configuration of behavior algorithm flowchart (number of loops, branching, logic of diagnostics system using - after diagnostics procedure RECS can restore its operating state or interrupt the performance of its objective function).

This behavior algorithm includes one loop which repeats algorithm's fragment of target searching and consists of 8 operating units (4 of them are ending units) and 5 conditional units (4 stochastic alternative units and 1 deterministic switching unit).

The scheme of paths of the RECS for searching, detection and locking-on targets consists of 5 functional units (one of them is repeated functional unit) (Fig. 1). Researching of the efficiency indexes of this complex system was carried out by developed model using Eq. 1, 2.

Firstly, increasing of the probability of successful performance $\Delta \mathrm{p}\left(\mathrm{S}_{\mathrm{SP}}\right)$ of RECS relative to initial probability of successful performance (probability of target searching $\mathrm{p}_{2}=0.9$ ) was carried out. Obtained graph (Fig. 2) shows that for given configuration of 
behavior algorithm the implementation of procedures for increasing the probability of target detection higher than 0.999 is impractical because the probability of successful performance of RECS objective function differs slightly.

Secondary, increasing of the probability of successful performance $\Delta \mathrm{p}\left(\mathrm{S}_{\mathrm{SP}}\right)$ of RECS relative to preceding probability of successful performance $\mathrm{p}\left(\mathrm{S}_{\mathrm{SP}}\right)$ as dependence of probability of target searching $\mathrm{p}_{2}$ is presented in Fig. 3.

Carried out research also shows that the implementation of procedures for increasing the probability of target detection higher than 0.999 does not significantly increase the probability of successful performance of RECS objective function.

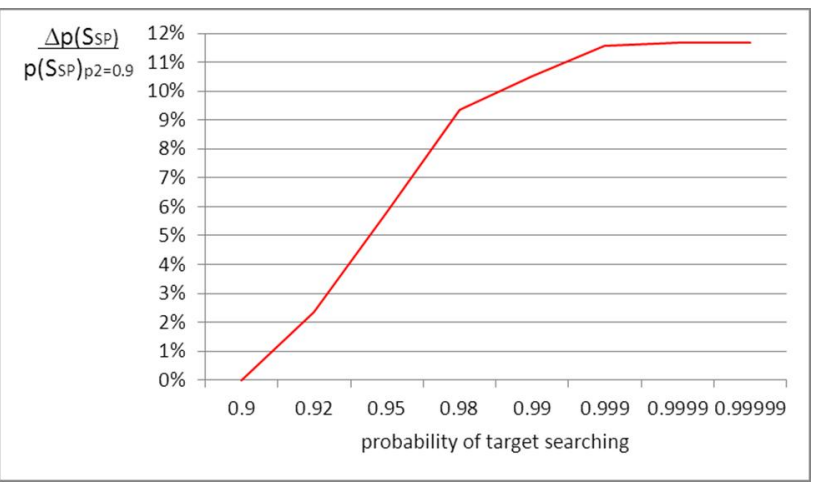

Fig. 2 - Increasing of probability of successful performance $\Delta p\left(\mathbf{S}_{\mathrm{SP}}\right)$ of $\mathbf{R E C S}$ relative to initial probability of its successful performance as dependence of probability of target searching $p_{2}$.

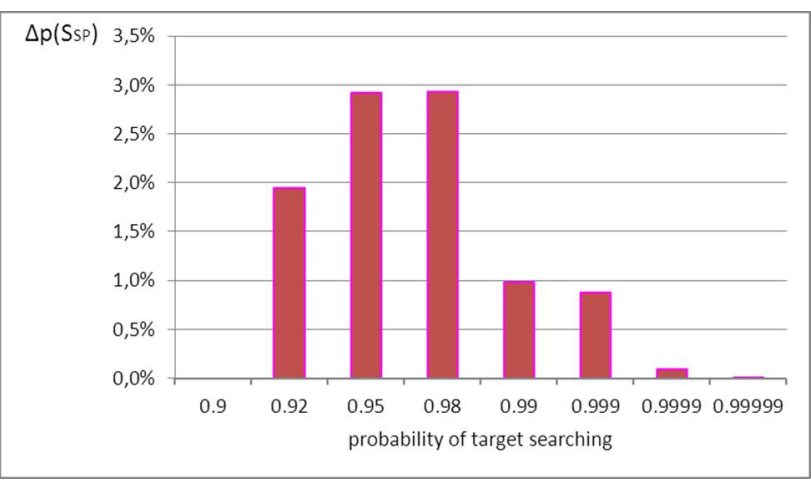

Fig. 3 - Increasing of probability of successful performance $\Delta p\left(S_{S P}\right)$ of RECS relative to preceding probability of its successful performance $p\left(S_{S P}\right)$ as dependence of probability of target searching $p_{2}$.

In third case the increasing of the probability of successful performance $\Delta \mathrm{p}\left(\mathrm{S}_{\mathrm{SP}}\right)$ of RECS relative to preceding probability of successful performance $\mathrm{p}\left(\mathrm{S}_{\mathrm{SP}}\right)$ as dependence of loops number $\mathrm{n}$ of behavior algorithm was conducted.

From this study (Fig. 4) it can be concluded that increasing the loops number greater than 2 in given behavior algorithm has practically no effect on the increasing of the probability of successful performance of the RECS objective function.

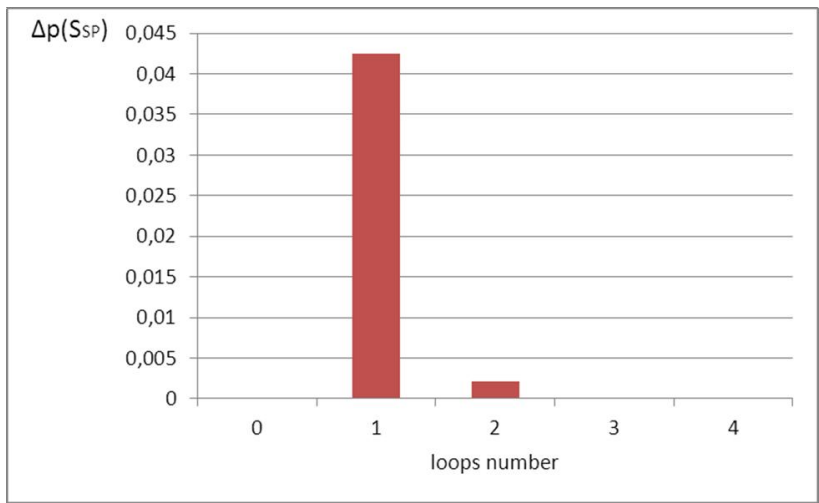

Fig. 4 - Increasing of probability of successful performance $\Delta p\left(S_{S P}\right)$ of RECS relative to preceding probability of its successful performance $p\left(S_{S P}\right)$ as dependence of behavior algorithm loops number $n$.

At the last, research of increasing of mean time value of successful performance $\Delta \mathrm{T}\left(\mathrm{S}_{\mathrm{SP}}\right)$ of RECS relative to initial mean time value of successful performance (loops number $\mathrm{n}=0$ ) as dependence of loops number $\mathrm{n}$ of behavior algorithm was completed.

Obtained graph (Fig. 5) shows that mean time value of successful performance of given behavior algorithm of RECS does not practically increase because of normalization and very low probability of loops performance when $\mathrm{n}$ is greater than 2 .

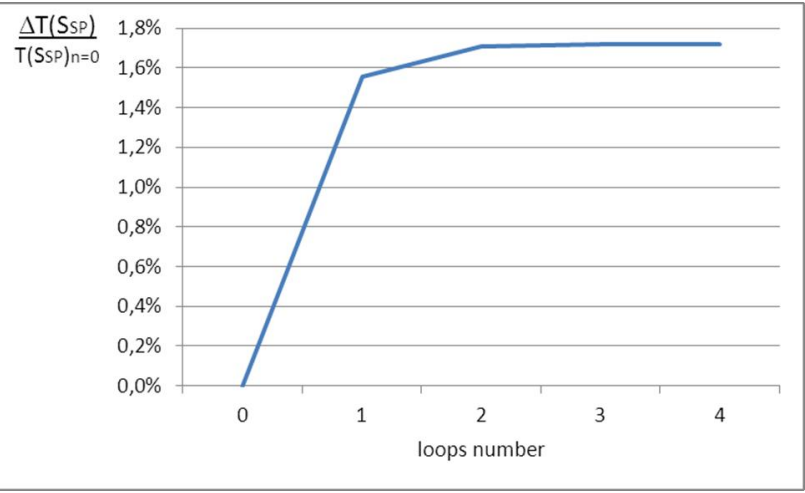

Fig. 5 - Increasing of mean time value of successful performance $\Delta T\left(S_{S P}\right)$ of RECS relative to its initial mean time value of successful performance as dependence of behavior algorithm loops number $\mathbf{n}$.

\section{CONCLUSION}

The new scheme of paths method for efficiency indexes estimation for behavior algorithm of RECS was developed. This new method takes into account all features of the behavior algorithms of RECS.

The scheme of paths is easily and directly formed from flowchart of behavior algorithm of RECS, that reduces time and costs for analysis of RECS at the 
design stage. Moreover, formalization and automation of the scheme of paths method were completed and tools for multivariate analysis of the effectiveness of the behavior algorithm of RECS were put in practice.

Also, as we demonstrated in paper [1], the model of behavior algorithm, which was constructed using scheme of paths method, gives commensurate values of efficiency indexes in comparison with mathematical models of the same behavior algorithm, which were obtained by the space of states and algorithmic algebras methods.

Given the above, we can conclude that, when multivariate analysis is required for design the behavior algorithms of RECS, the scheme of paths method has advantages in comparison with known methods.

\section{REFERENCES}

[1] B. Yu. Volochiy, L. D. Ozirkovskyi, O. P. Shkiliuk, A. V. Mashchak, Method of Efficiency analysis of behavior algorithms of radio electronic complex system for critical applications, Scientific and Technical Magazine "Radioelecrtonic and Computer Systems" (70) 6 (2014), pp. 130-134. (in Ukrainian)

[2] A. S. Mozhaev, General Logical-Probabilistic Method of Reliability Analysis of Complex Systems, VMA Publishing House, Leningrad, 1988, 63 p. (in Russian)

[3] A. S. Mozhaev, V. N. Gromov, Theoretical Foundations of General Logical-Probabilistic Method of Automatic Modeling of Systems, VITU Publishing House, Sankt-Peterburgh, 2000, 145 p. (in Russian)

[4] J. Magott, P. Skrobanek, Timing analysis of safety properties using fault trees with time dependencies and timed state-charts, Reliability Engineering and System Safety, (97) (2012), pp. 14-26.

[5] A. Soltani Tehrani, Behavioral Modeling of Radio Frequency Transmitters, Chalmers Reproservice, Göteborg, 2009, 63 p.

[6] J. Svobodová, Parallel Computing and Neural Networks in Behavioral Modeling, Brno University of Technology, Brno, 2012, 62 p.

[7] H. Pham, Reliability Modeling, Analysis and Optimization, World Scientific Publishing Co., Singapore, 2006, $487 \mathrm{p}$.

[8] I. Koren, C. Mani Krishna, Fault Tolerant Systems, Elsevier / Morgan Kaufmann Publishers, San Francisco, 2007, 387 p.

[9] B. Yu. Volochiy, Modeling Technology of Behavior Algorithms of Information Systems. Lviv Polytechnic National University
Publishing House, Lviv, 2004, 220 p. (in Ukrainian)

[10] B. Yu. Volochiy, L. D. Ozirkovskyi, O. P. Shkiliuk, A. V. Mashchak, Method of estimation of indexes of efficiency of the radio electronic complex system monitoring of air space, Bulletin of Lviv Polytechnic National University, (766) (2013), pp. 192-201. (in Ukrainian)

[11] I. V. Safonov, Reliability Engineering of Control Algorithms. Far East Scientific Centre Publishing House, Vladivostok, 1982, 156 p. (in Russian)

[12] J. L. Peterson, Petri Net Theory and the Modeling of Systems, Prentice Hall, Englewood Cliffs, 1981, $290 \mathrm{p}$.

[13] M. Boualin, P. Barger, W. Schon, Backward reachability of colored Petri Nets for systems diagnosis, Reliability Engineering and System Safety, (99) (2012), pp. 1-14.

[14] N. Sadou, H. Demmou, Reliability analysis of discrete event dynamic systems with Petri Nets, Reliability Engineering and System Safety, (94) (2009), pp. 1848-1861.

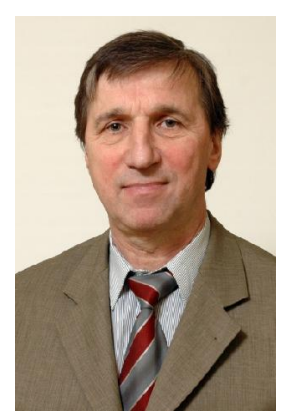

Bohdan Volochiy, Dr.Sc., Professor of Theoretical Radio Engineering and Radio Measuring Department of Lviv Polytechnic National University. Experience of teaching in higher education for is over 35 years. Author of 160 publications, including 3 monographs, 3 textbooks, 4 inventions.

Research interests are: theory and practice of system design of radioelectronic information systems.

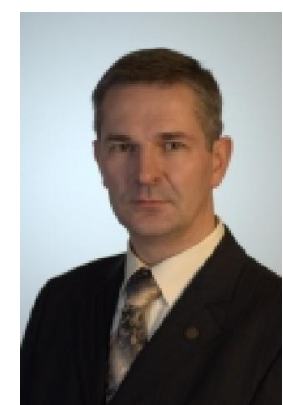

Leonid Ozirkovskyi, Ph.D., Associate Professor of Theoretical Radio Engineering and Radio Measuring Department of Lviv Polytechnic National University. His teaching experience in higher education has been developing for over 10 years. The author of 72 scientific publications and 26 scientific and methodical works, including 1 mono-graph, 4 textbooks. He has prepared 1 candidate (PhD) of technical sciences. Research interests include the development of methods and tools for modeling functional and reliability behavior of information systems. 


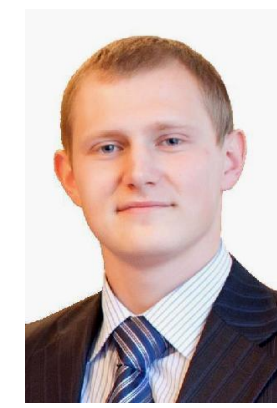

Oleksandr Shkiliuk, postgraduate of Theoretical Radio Engineering and Radio Measuring Department of Lviv Polytechnic National University. Research interests are: methods, techniques and tools for construction models for reliability analysis of behavior algorithms of radio electronic complex systems.

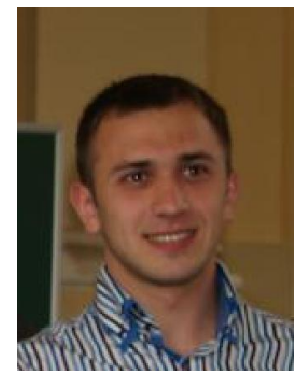

Mashchak Andriy, postgraduate of Theoretical Radio Engineering and Radio Measuring Department of Lviv Polytechnic National University. Research interests are: methods, techniques and tools for ensuring reliability and safety of radio electronic complex systems. 